\title{
Espacialidad y dominación del proyecto saudí NEOM: espejismo de desarrollo y sustentabilidad
}

María Fernanda Correa Alejandri *

\section{Resumen}

$\mathrm{C}^{1 \text { Reino de Arabia Saudita (RAS) implementó en } 2017 \text { un pro- }}$ Cyecto denominado Visión 2030, el cual posee como objetivo esencial la diversificación del mercado saudí y el desarrollo económico de la nación. A través de dichas reformas estructurales se desprende NEOM, una megaciudad con tintes futuristas, la cual no estará regida bajo los preceptos islámicos y de la que se esperan importantes ventajas económicas. En el presente artículo se aborda la construcción de este proyecto a través de los intereses geopolíticos que persigue, así como el impacto espacial que de él se derivan: dinámicas de despojo, desplazamiento y una distribución desigual de la riqueza. Con lo anterior, se propone cuestionar su viabilidad en términos energéticos y sociales.

\section{Palabras clave}

Reino de Arabia Saudita, producción del espacio, modernidad capitalista, geopolítica, seguridad energética, energías renovables.

Fecha de recepción: marzo de 2021
Fecha de aceptación: junio de 2021

Licenciada en Relaciones Internacionales, por la Facultad de Ciencias Políticas y Sociales en la Universidad Nacional Autónoma de México. Asesor: Mtro. Irwing Rico. (FCPyS UNAM) Contacto: alejandrifer-nanda98@gmail.com ORCID: https://orcid.org/0000-0001-6540-5488 


\title{
Spatiality and Domination of the Saudi NEOM Project: Mirage of Development and Sustainability
}

\author{
Keywords \\ Kingdom of Saudi Arabia, spatial production, modernity, capitalism, \\ geopolitics, energy security, renewable energy sources.
}

\section{Abstract:}

The Kingdom of Saudi Arabia implemented in 2017 a project called Vision 2030, which its main scope is the diversification of the Saudi market and its economic development. Consequently, NEOM emerged as a megacity with futuristic goals, which will not be under Islamic precepts and rules and from which it expects significant economic advantages. This paper focuses on the construction of NEOM's project, from the geopolitical interests it pursues and the spatial impact derived from it: dispossession, displacement, and unequal distribution of wealth. Moreover, our purpose is to question its viability in energy and social terms.

\section{Introducción}

El sistema capitalista basa sus cimientos en el flujo constante de capital, que se materializa en la acumulación, inversión e intercambio, expresados a través de alianzas geoestratégicas en términos políticos, sociales y económicos. Bajo esta línea, la construcción de los llamados megaproyectos representan una vía para lo anterior, los cuales, bajo estandartes de desarrollo y progreso, permiten la edificación de grandes estructuras urbanas supuestamente modernas que prometen bienestar y crecimiento económicos a la población, y que resultan estratégicos para el actual modo civilizatorio.

Con el objetivo de mantener su hegemonía regional el Reino de Arabia Saudita (RAS) ha buscado implementar estrategias que le permitan afirmarse e innovarse, todo en un contexto de superación del llamado capitalismo fósil. La posición geográfica del RAS le ofrece ventajas enormes sobre el petróleo, ya que su facilidad 
de alcance y extracción permiten que se posicione dentro de los cinco países más ricos de la región con un PIB de alrededor de \$792, 967 millones de dólares, de acuerdo con datos del Banco Mundial (2019). Sin embargo, a raíz del calentamiento global, al cada vez más próximo agotamiento de recursos petrolíferos y en el marco de la agenda sostenible, el país asiático se ha visto obligado a tomar nuevas medidas.

En este sentido, Visión 2030 es un plan de transformación nacional para reducir la dependencia de Arabia Saudita del petróleo, diversificar su economía y desarrollar sectores de servicios públicos. Entre los objetivos más importantes se incluyen el refuerzo de las actividades económicas y de inversión, el aumento del comercio de la industria no petrolera, el aumento del gasto gubernamental en el ámbito militar y la construcción de una megaciudad con jurisdicción propia.

Llamada Ciudad NEOM, se trata de una de las urbes inteligentes que se vende como la más moderna en términos tecnológicos y sociales. Esta ciudad inteligente promete un impulso regional y una proyección internacional bastante ambiciosas. Además de las metas económicas, representaría un modelo a seguir en el contexto de superación y mitigación del cambio climático, al priorizar la implementación de las energías renovables como la solar y la eólica. No obstante, este proyecto perpetúa un modelo civilizatorio específico, el cual realmente no proporciona soluciones viables a las tantas contradicciones que el sistema capitalista ha ocasionado.

Por lo anterior, el presente trabajo argumenta que la Ciudad NEOM en realidad no es una alternativa, sino que simboliza la misma promesa de modernidad-capitalista en términos de homogeneización. De esta manera, el siguiente análisis aborda en un primer momento el eje conceptual, con el fin de profundizar en el proceso de producción de un espacio en donde se llevan a cabo situaciones de despojo, alienación e igualación, con el objetivo de garantizar que el capital altere todo ámbito de la realidad. De manera siguiente, se procederá a ahondar en la edificación del Proyecto NEOM, tomando en cuenta las situaciones sociales, políticas, 
económicas y geoestratégicas que implica para que, finalmente, se cuestione la viabilidad y objetivo verdaderos de dicho proyecto.

\section{Fundamentos del desarrollismo capitalista-moderno}

La modernidad impone ciertos supuestos epistemológicos, ontológicos, y filosofías que priorizan la razón y el progreso de la humanidad a cualquier coste. Se trata de un proceso civilizatorio que se instaura en todo el sistema mundial y, al tener carácter homogeneizante, es aceptada de manera automática sin importar qué tan incongruente, insostenible o dañino sea. Este matiz ha sido atribuido por la estructura capitalista en la cual vivimos, generando categorías para asegurar una valoración utilitaria e instrumental de la realidad. Además de ello, el capitalismo tiene como característica el ser exponencial, intensivo y expansivo, por lo que encontrar nuevas formas de apropiación, espacialidad y dominio es vital para entender su voracidad.

Uno de los bastiones más importantes que permite su materialidad es el de desarrollo, un discurso y modelo civilizatorio construido históricamente con el objetivo de moldear al mundo. Consiste en la adopción de ideologías, formas de organización político-económicas, y abstracciones que permiten una articulación social, la cual tiene como objetivo establecer un estilo de vida congruente con el sistema hegemónico occidental. Esto permite que, casi por inercia, individuos, sociedades, países y la humanidad en su totalidad se vean abrumados por una disyuntiva: el de subirse al tren de la prosperidad y mejora, o ser relegados en la barbarie e incultura:

[...] el discurso del desarrollo [...] ha producido un aparato muy eficiente para producir conocimiento [...] Dicho aparato surgió en el periodo comprendido entre 1945 y 1955 , y desde entonces no ha cesado de producir nuevas modalidades de conocimiento y poder, nuevas prácticas, teorías, estrategias, y así sucesivamente. En resumen, ha desplegado con buen éxito un régimen de gobierno [...] (Escobar, 2014). 
La connotación económica de esta narrativa resulta imprescindible para el crecimiento del capitalismo, característica sine qua non de su funcionamiento. Ello ha derivado, en primer lugar, en la construcción de una imaginación sociológica que, a partir de categorías como "pobreza", "penuria" o "atraso", permite crear un estandar y legitimar la dominación de culturas históricamente infantilizadas. Por otro lado, el desarrollismo también ha logrado configurar una cartografía dividida en centros y periferias que, en el marco de un mismo circuito productivo, genera sociabilidades de explotación y dependencia hacia los centros. De esta manera, "esta producción social del espacio está ligada a la producción de diferencias, subjetivididades y órdenes sociales” (Escobar, 2014).

Bajo esta tesitura, y de acuerdo con los planteamientos de Henri Lefebvre (1974), el capitalismo requiere de la producción de espacios que posibiliten su continuidad y serán estas espacialidades mediante las cuáles se domine. El surgimiento de estos espacios dominantes implica despojo y violencia, teniendo afectaciones en aspectos ambientales y sociales en la forma de organización y vivienda de las diferentes comunidades, lo que resulta en una gran paradoja o lo que podría denominarse como la "tragedia fáustica de desarrollo" (Berman, 2004). ${ }^{1}$

En este sentido, es necesario categorizar y determinar lo bueno y malo para el sistema. Con ello, la construcción de la seguridad supone algo esencial para el mantenimiento del status quo. Es imperativo que exista un ordenamiento para garantizar cierta gubernamentalidad, por lo que el espacio conlleva relaciones de poder y dominación con el fin de cumplir intereses específicos. Es decir, bajo el pretexto del desarrollo y modernidad el capital busca garantizar que su flujo nunca se agote, procurando la eliminación

\footnotetext{
1 Consultar Marshall Berman, (2004) en Todo lo sólido se desvanece en el aire. La experiencia de la modernidad, con base en una alegoría a la obra de Johann Wolfgang von Goethe, "Fausto", quien representa una modernidad o espíritu insaciable que no se contenta con la realidad, hace referencia a que la única manera para transformar al mundo es hacerlo en su totalidad, en términos sociales, morales y humanos. Estos grandes desarrollos terminan por exigir grandes costes; el abandono al mundo anterior pre-capitalista o tradición; la materialidad y el ser del humano creativo; y la destrucción del medio ambiente.
} 
de todo aquel individuo, situación o fundamento que signifique su invalidez. Esto crea un ambiente de guerra constante, el cual es expresado como forma de reproducción de una hegemonía.

Asimismo, es importante destacar que el desarrollo va a irse adecuando a la coyuntura del orden internacional. Las contradicciones del sistema capitalista han generado una amplia gama de problemáticas como la desigualdad, empobrecimiento, crisis financieras y, quizá la más importante, el calentamiento global. Su superación, amortiguación y, en el peor de los casos, adaptación al mismo, son retos importantes del siglo XXI a los que se enfrenta la sociedad internacional y representan la razón perfecta para perseguir la meta primordial: el desarrollo en términos "sustentables, justos y benéficos".

Estas alternativas, si bien a primera vista podrían parecer la solución adecuada, suponen meras ideas autocomplacientes de la modernidad, que confían en la economía y en la tecnología para lidiar con todas las dificultades que la humanidad ha perpetrado para sí misma. En otras palabras, se trata de una modernidad reflexiva proveniente de una sociedad que hace todo lo posible para prever y controlar los riesgos existentes, ${ }^{2}$ todo con el fin de seguir sustentando la misma racionalidad de vida. ${ }^{3}$

2 Ulrich Beck, (1998) en La Sociedad del Riesgo. Hacia una nueva modernidad, realiza aportes sustanciales respecto a cómo se ha gestado un entorno altamente peligroso para la existencia de la misma humanidad, derivado de los ideales de progreso, desarrollo e innovación propios de la modernidad. Al ser conscientes de los daños y a través de una "modernidad reflexiva", esta sociedad hace todo lo posible para prever y controlar estos riesgos, todo con el fin de seguir sustentando una sociabilización específica. Por tanto, el miedo se posiciona como eje de estructuración y la incertidumbre predomina en las dinámicas sociales.

3 Enrique Leff (2010) en Imaginarios sociales y sustentabilidad, se cuestiona el concepto de modernidad reflexiva argumentando que se trata de una reflexión ficticia que continúa priorizando la razón modernizante. Al no cuestionar principios, ideologías y saberes, no representa una solución viable en contraposición con la promoción de una racionalidad ambiental. 
Ciudad NEOM: implicaciones económicas y geopolíticas del proyecto del futuro

El desarrollo del proyecto NEOM se enmarca en Saudi Vision 2030, un plan estructural dirigido por el príncipe Mohammed bin Salmán, que busca lograr a través de una serie de reformas la independencia económica del sector petrolero en que se encuentra inmersa el RAS y del cual en 2019 se obtuvieron ingresos de más de 594.4 miles de millones de riales (Saudi Arabia Monetary Agency, 2019). De esta forma, a través de un bastión vanguardista, la visión saudí planea ser reconocida como el corazón del mundo árabe e islámico, inmerso en la llamada península arábiga.

Un segundo y tercer pilar de esta nueva visión se enfocan en la determinación de convertirse en una potencia internacional y transformar al país y región en un centro global que conectaría tres continentes: Asia, Europa y África. Lo anterior, subsidiado mediante la transformación del fondo de inversión público en el mayor fondo soberano de inversión, es decir, a través de la inversión directa de la corona real auxiliada por la entrada de capital empresarial. De acuerdo con el Saudi Arabia Monetary Agency:

El Fondo de Inversión Pública (FIP) busca mejorar su papel en la aceleración de la transformación económica nacional [...] Con el fin de lograr sus objetivos de manera eficiente y maximizar las ganancias a obtener, en concordancia con Saudi Vision 2030, el FIP tiene como objetivo diversificar sus fuentes de ingresos ampliando sus inversiones internacionales, entablando asociaciones estratégicas y promoviendo importantes iniciativas. Entre los logros más destacados del FIP durante 2019 fue el crecimiento de su inversión de cartera, impulsando el valor total de sus activos hasta 1.2 billones de riales (SAMA, 2019).

Aunado a ello, es necesario señalar que dicha reestructuración trae consigo planes fiscales de balance, regulaciones y programas de revisión, mediciones en cuanto al desempeño, así como un apartado especial destinado a Saudi Aramco, una de las empresas 
petroleras más importantes del país y de la cual se busca diversificar su campo de acción y no solo restringirla al marco petrolero, de acuerdo con la corona saudí. La principal medida para cumplir con los objetivos de Vision 2030 es su privatización, ya que se trata de la empresa más rentable del mundo. En 2018 generó \$111 mil millones de dólares en ingreso neto y $\$ 86$ mil millones de dólares en flujo de efectivo disponible (Holmes, 2019).

Alrededor del valor total de la empresa han circulado debates. Muhammad bin Salmán ha llegado a sugerir que vale $\$ 2$ billones de dólares; de esta forma, la venta de un 5\% de las acciones significaría una entrada de efectivo de $\$ 100$ mil millones de dólares. (Henderson: 2019) Por ello, el 3 de noviembre de 2019 la empresa petrolera anunció su intención de cotizar acciones en la bolsa de valores local, con el objetivo de diversificarse y hacer de la empresa más competitiva en diversos sectores energéticos, así como obtener más recursos para financiar el proyecto tan ambicioso que supone lo plasmado en Visión 2030.

Los resultados que se esperan a través de este proyecto son ambiciosos y de ellos puede desprenderse la creación de megaproyectos que auxilien a la entrada de capital financiero. Tal es el caso de la visión futurista plasmada en NEOM, la cual nace del deseo de Arabia Saudita por ver al país convertirse en un modelo pionero y próspero en varias e importantes áreas, y que tiene como objetivo impulsar la transformación del Reino en un centro global a través de la introducción de cadenas de valor industriales y tecnológicas. (Fondo de Inversión Pública de Arabia Saudita, 2017).

NEOM busca ser una de las megaciudades más importantes conocidas hasta el día de hoy. ${ }^{4}$ La posición geográfica en la que se

\footnotetext{
4 Para lograr dicho objetivo y en concordancia con lo plasmado en Vision 2030, es importante mencionar que la ciudad NEOM no estará regida bajo los preceptos islámicos. Esto quiere decir que los Ulema - expertos en asuntos jurídico-religiosos del islam sunni-, no tendrán jurisdicción sobre la urbe, por lo que no seguirá las reglas y regulaciones aplicadas en el resto de Arabia Saudita, en donde se aplica la ley sharia basada en la interpretación wahabi del islam. De esta forma, ofrecerá a los residentes un estilo de vida más "liberal" y occidental, permitiendo actividades como conciertos, el consumo de alcohol, la convivencia pública entre géneros, o la libre elección de vestimenta, por mencionar algunos ejemplos.
} 
ubica este megaproyecto ha sido una de las bases más fuertes que sustentan su construcción. El espacio elegido consta de unos 100 kilómetros de costa virgen situados al norte del golfo de Ácaba con $26.500 \mathrm{~km}^{2}$ en total. Su localización en una zona limítrofe con Egipto y Jordania; próximo a Israel. Fue optado con el fin de que pudiera integrarse a un área turística colindante con Jerusalén, la Península del Sinaí y Petra. De acuerdo con datos oficiales:

La región se encuentra en la encrucijada del mundo: el 40 por ciento de la población mundial podrá llegar a NEOM en menos de cuatro horas; aproximadamente el 13\% del comercio mundial ya fluye a través del Mar Rojo. Volando al aeropuerto de NEOM, Londres está a solo cinco horas de distancia, Dubai y El Cairo a dos, y Zurich a cinco y media (NEOM, 2021).

Con el uso de la tecnología, la energía sustentable y la serie de recursos obtenidos en la región, aunado a su importante posición estratégica, el proyecto se convierte en un anhelo por diversificar la entrada de capital económico extranjero, comenzando con reformas de carácter neoliberal que buscan generar ganancias entre la élite saudí. Inmersos dentro del llamado capitalismo verde y con un presupuesto de más de $\$ 500$ mil millones de dólares, Visión 2030 y el Proyecto NEOM en específico, buscan construir un espacio que suponga un mejor estilo de vida que aquel visto en las ciudades actuales más modernas en términos sociales, ecológicos y económicos.

En este sentido, un nuevo modelo de sostenibilidad urbana, protección al medio ambiente y el uso eficaz y productivo de la tecnología son los ejes angulares del desarrollo de dicho proyecto. Para ello, el Programa Nacional de Energías Renovables, supervisado por el Ministerio de Energía, Industria y Recursos Minerales del Reino es una iniciativa estratégica a largo plazo que apoya directamente la Visión 2030. Este programa no solo reduciría la dependencia petrolera, así como las emisiones de gases de efecto invernadero como parte del Acuerdo de París; sino que también permitiría la creación de empleo y promovería el desarrollo económico en el Reino. 
En particular, en esta línea resulta relevante la firma de un acuerdo para la construcción de una planta de producción de amoníaco a base de hidrógeno verde a escala mundial, alimentada por energía renovable con valor de $\$ 5$ mil millones de dólares (Matthew, 2021). El proyecto, firmado entre Air Products junto con ACWA Power, sería propiedad a partes iguales de los tres involucrados; estaría situado en NEOM y produciría amoníaco verde para exportar a mercados globales. El acuerdo fue firmado el 20 de julio de 2020 y esta sería la primera asociación para NEOM con socios líderes en el campo de la energía renovable. Es un elemento fundamental para su estrategia en volverse un actor importante en el mercado global de hidrógeno, así como un propulsor de megaestructuras que pretendan transitar hacia un nuevo régimen energético.

Asimismo, y con referencia al marco estratégico que representa el proyecto en términos de seguridad y expansión, es importante señalar las alianzas establecidas con el objetivo de ampliar mayores canales de entrada hacia la ciudad y conectar, no solo las ciudades más importantes, sino generar vínculos de comunicación con otros Estados. En el primer caso se encuentran países como Egipto e Israel, y en el parámetro de distensión existen ejemplos como el de Irán.

Lo que concierne al caso egipcio puede clasificarse como una alianza que producirá beneficios para ambos Estados, pues no es menor recordar que existen antecedentes de negociación con el objetivo de conectar ambos países. De acuerdo con Moisés Garduño (2020), el ejemplo claro de lo anterior es la donación de las islas Tirán y Sanafir por parte del gobierno egipcio para la construcción del puente King Abdel Aziz en manos del gobierno saudí: "En el caso de El Cairo se trata de un aliado clave en tanto NEOM necesita mano de obra para ser construida, necesita la herencia egipcia como atracción principal de turistas a nivel mundial y, particularmente, necesita infraestructura para mejorar la seguridad de las fronteras" (Garduño, 2020, p. 150).

A partir de un somero análisis por medio de los intereses de ambos Estados, el proyecto NEOM vino a propiciar un mayor 
acercamiento entre los mismos, puesto que la frágil economía egipcia golpeada en los últimos años requiere inversiones de este carácter para ser soliviantada, aunque ello genere una mayor dependencia económica. En el caso del Estado saudí, Egipto representa una importante zona de seguridad e influencia a través del Golfo Pérsico y el Mar Rojo.

Ello podría ser considerado como un acuerdo de seguridad sostenible. Lo anterior, debido a que este tipo de negociaciones se convierten en el pretexto perfecto para permitir la transacción de grandes cantidades de dinero con la finalidad de estabilizar el Estado egipcio y, de igual manera, permitir la securitización ${ }^{5}$ de la zona a través de la compra de armas.

Por su parte, lo que representa el proyecto para generar alianzas con otros Estados, destaca el caso de Israel. Con dicha nación se pretende que, a través del apoyo egipcio, pueda entablarse un acuerdo de seguridad regional. Desde hace varios años las relaciones diplomáticas entre ambos países se han estrechado debido a un común objetivo, la contención iraní; mientras que para Israel, la problemática principal es el no reconocimiento estatal por parte de Irán, para Arabia Saudita el obstáculo radica en el tipo de Islam chí́ta revolucionario del régimen iraní, el cual, rechaza el orden monárquico. Por otro lado, este acercamiento también se ha dado debido al surgimiento de grupos salafistas sunitas como ISIS y la Hermandad Musulmana, y a la activa y asertiva participación del RAS en las negociaciones israelí-palestinas (Rynhold \& Yaari, 2019).

Ahora bien, los verdaderos intereses israelíes de apoyar la creación de un megaproyecto de esta índole radican esencialmente en que, con un campo de acción mayormente liderado por el RAS, el estado bélico que posee la región puede perpetuarse; lo cual es traducido como inversiones en armamento, mismo que

5 Acuñado por Ole Waever (1995), se trata de un concepto que alude a a la construcción discursiva de ciertos temas para incorporarlos como amenazas a la seguridad, es decir, la "creación" del enemigo, así como el engranaje político, social y cultural en torno a él. Se refiere sobretodo a la forma mediante la cual ciertos actores construyen una narrativa sobre la existencia de supuestas amenazas. 
ha llegado a especularse podría ser de carácter nuclear. Si bien es cierto que NEOM es un megaproyecto que ofrece fuertes virtudes económicas a largo plazo y plantea estar apoyado de la sustentabilidad, la realidad es que detrás de la ciudad futurista existe un interés geopolítico y estratégico que busca ampliar las influencias del RAS hacia determinadas zonas de interés.

\section{Verdadera viabilidad: despojo, geoestrategia y la élite dominante}

De acuerdo con datos del Institut de Publique Sondage d'Opinion Secteur, el anuncio del proyecto NEOM fue recibido de muy diversas maneras. La mayoría de la población saudí cree que la urbe inteligente será un motivo de orgullo para los nacionales, mejorando la imagen del Reino en todo el mundo, además de que se espera que abra puertas para nuevas oportunidades laborales. A pesar de que el RAS cuenta con uno de los índices económicos más altos de la península arábiga, el anhelo por parte de la población por conseguir nuevas vías de empleo, a primera vista podría ser un indicador de una mala distribución de riqueza. Esto deriva de graves acusaciones de corrupción contra la corona saudí, la cual anteriormente ha realizado gastos exorbitantes con el único fin de obtener ocio y placer.

Así, con un porcentaje de desempleo del 5.8\% su aceptación casi ciega posiciona a NEOM como una de las alternativas más apoyadas a nivel local. Por otro lado, las mayores preocupaciones y negativas - mostradas por únicamente $10 \%$ de la población-, estaban relacionadas con la finalización e implantación del proyecto, junto con las inquietudes sobre cómo NEOM afectará la identidad cultural y religiosa de Arabia Saudita. Entre otras críticas respecto a la mega urbe, los habitantes saudíes se muestran consternados respecto de su alto costo, el posible aumento de impuestos, un incremento en el costo de vida, falta de empleo para los ciudadanos, alta dependencia tecnológica y un probable aumento de discriminación racial. 
Asimismo, las comunidades locales mostraron preocupación por sus tierras desde el anuncio del proyecto urbano en abril de 2017, debido a fuertes rumores de desalojos. Estos temores se hicieron realidad en enero de 2020, cuando las autoridades locales informaron a los habitantes de la decisión de desalojarlos. En un primer momento, esta orden de desalojo fue recibida de manera pacífica por la oposición local, en su mayoría miembros de la tribu Huwaitat, que tiene importante presencia en el suroeste de Jordania, el Sinaí de Egipto y el noroeste de Arabia Saudita. Sin embargo, la postura de las autoridades del gobierno saudí fue en su lugar violenta y abusiva (Middle East Eye, 2020).

Esto demuestra que la construcción de dicha idea, pese al discurso de mejora para la región y el país, afecta directamente la condición de vida de las poblaciones aledañas. Las comunidades nativas sufren del arrebato de la tierra en donde llevan a cabo sus actividades diarias y que son indispensables para su bienestar y su cultura. Bajo esta óptica, el papel del gobierno no es procurar a su población, sino más bien, afirmar su proyección regional, nacional e internacional. Esto responde a la misma naturaleza del sistema y modo de producción, el cual, basa sus cimientos en indicadores artificiales como el PIB o el IPC que no tienen otro objetivo más que fetichizar el crecimiento y mercantilizar el modo de vida.

Se confirma entonces que a través de discursos modernizantes el Estado saudí se excusa y se autoriza para consolidar dos aspectos fundamentales en el sistema capitalista imperante. En primer lugar, la cuestión del control de los espacios que posibilitan la extracción de recursos y con ello, el ficticio enriquecimiento de las naciones y, posteriormente, la habitual lógica de enmarcar estratégicamente dichas espacialidades a favor de una realidad impuesta sistémicamente, en el cual, distintas formas de habitar, pensar y vivir son repelidas.

Así, tras la reacción violenta por parte del gobierno, se realizaron numerosos intentos por mantener ocultas las posturas de dichas comunidades del conocimiento público. Asimismo, numerosas violaciones tuvieron lugar con el propósito de controlar a las voces principales de la tribu Huwaitat; 
[...] en el contexto de una represión más amplia de las libertades cívicas y disidencia pacífica en Arabia Saudita, que se ha intensificado en los últimos años, incluyendo fuertes intimidaciones, arrestos arbitrarios, detenciones prolongadas, torturas brutales y organizando ejecuciones; silenciando cualquier voz que se pronunciara (Habitat International Coalition, 2020).

La situación se ha agravado derivado del asesinato del activista Abdul-Rahim al-Howeiti, miembro tribal que protestaba por su desalojo. Su viralización se debe a un video publicado en las redes sociales, en el cual Howeiti documentó sus momentos finales antes de que se le disparara, mostrando el área de la que sus vecinos fueron sacados por la fuerza. El activista grabó imágenes desde su techo, mostrando a decenas de fuerzas de seguridad disparando mientras patrullaban el área. En el video, Howeiti también manifiesta:

La gente está siendo eliminada de sus hogares y la gente no está de acuerdo en absoluto con lo que está sucediendo. Pero la forma en que el Estado ha manejado las cosas solo puede describirse como terrorismo... terrorismo de Estado. [...] Estoy en contra del traslado forzoso de personas. No quiero irme, quiero quedarme en mi casa. No quiero compensación, no quiero nada. [...] La sensación generalizada aquí es que la gente se opone y ninguna compensación bastará, incluso si se les paga 100 millones de riales (Middle East Eye, 2020).

Por naturaleza, la construcción de un espacio dominante produce relaciones de subordinación y sometimiento. El desarrollo, por la misma naturaleza del sistema capitalista, así como por meras limitaciones físicas, no puede ser para todos. Por consiguiente, el despojo resulta necesario. A ello hay que sumarle la validez y legitimación que recibe por parte del Estado, el cual evidentemente para procurar el flujo de capital y, por ende mejorar sus condiciones económicas, debe garantizar las bases necesarias para su recibimiento apropiado. 
Una de las labores estatales es legitimar sus proyectos a través delas supuestas mejoras que representarían. En su contexto de dependencia fósil y el pronto agotamiento de dichos recursos, las energías renovables suponen para el RAS una milagrosa solución que le permitiría brindarle confianza a su población. La idea es tan ambiciosa, que es importante cuestionarse si las energías renovables podrán hacer frente al estilo de vida que NEOM está promoviendo. Su alta dependencia petrolera así como su posición geográfica y $s$ ituación c limatológica complejizan e l p roceso d e búsqueda de transición a energías renovables.

La generación de energía renovable del RAS para el 2020 fue de apenas 1.8 terawatts, lo cual contrasta diametralmente con su consumo de 3,788 miles de barriles de petróleo diarios, de acuerdo con datos de British Petroleum. Es cierto que la región tiene un alto potencial de energía solar, ya que la radiación promedio del país varía de 4 a 7 kilovatios. Pero, Arabia Saudita sigue generando más del $99 \%$ de su electricidad, utilizando petróleo y gas natural. Con este antecedente, la urbe se torna irrealizable, ya que al promover prácticamente el mismo estilo de vida consumista, no hay escenario posible para que de manera inesperada la demanda energética decrezca.

Muchos críticos han calificado a NEOM como un derroche, además de ser ineficaz para abordar los problemas de la economía saudí, como lo son el desempleo y su dependencia fósil. Estas opiniones se acentúan tomando en cuenta proyectos similares, como lo es Shenzhen en China y Silicon Valley en Estados Unidos. Estas Zonas Económicas Especiales se caracterizan por su cercanía a centros de industrias de alta tecnología, donde instituciones técnicas y sociales, desde el suministro de energía hasta las universidades intensivas en investigación, están firmemente establecidas (Demowski, 2018). Este tipo de entornos les permite lidiar con problemas imprevistos, algo que NEOM no parece tener mucho en cuenta.

Por ello, hay quienes afirman que NEOM es una especie de excusa para justificar las alianzas regionales que hacen frente a una posible nueva potencia regional: Irán. Esto se suma a un largo antecedente de fricciones entre ambos países cuyo o- 
rigen puede ubicarse desde el término de la Twin Pillar Policy ${ }^{6}$ estadounidense, con el surgimiento de la Revolución Iraní en 1979.

Acompañado de dicho país es necesario mencionar a aquellos contra los que hace frente el deseo expansionista saudí, y que en gran medida comparten ese interés en común como lo es el caso de Catar. Una de las respuestas para hacer frente a los posibles mecanismos de expansionismo de ambos han sido acuerdos como la Alianza contra el Terrorismo (ETIDAL) en diciembre del 2015, acuerdo mediante el cual se busca replantear el cumplimiento de la seguridad árabe. A través de este, el RAS acusó a Catar de tener prácticas en favor del terrorismo, realizando bloqueos económicos y diplomáticos apoyados por otros Estados de la región y que apenas en 2021 fueron revertidos (Garduño, 2020).

\section{Reflexiones finales}

NEOM es uno de los megaproyectos infraestructurales más ambiciosos de los últimos años. De acuerdo con el planteamiento espacial de Henri Lefebvre, estas grandes edificaciones resultan estratégicas para el sistema de producción imperante, produciendo un espacio específico que domina al sujeto a través de dinámicas de despojo e imposición de una materialidad instrumental que moldea las cotidianidades de los sujetos. Asimismo, permiten implantar ideologías, conductas condicionadas y un metabolismo social específicos que no solo garantizan los flujos del capital mediante supuestas inversiones e incremento de la fuerza laboral, sino que también otorgan al Estado Saudí una oportunidad para consolidarse geopolíticamente.

Ahora bien, a pesar de las ya mencionadas ventajas, es notorio que los resultados del proyecto se han visto obstaculizados. En primera instancia, destaca la resistencia por parte de la tribu

6 Política de doble contención que tenía como objetivo convertir a ambos países en "guardianes" de los intereses estadounidenses en la región. De esta manera, su fin principal era hacer frente a las tendencias panarabistas que comenzaban a surgir en esa época a través de financiamiento económico y armamentístico. 
Huwaitat junto con el asesinato de Abdul-Rahim al-Howeiti, los cuales debido a la divulgación por parte de medios de comunicación internacionales, ha generado un gran cuestionamiento sobre el actuar del gobierno saudí, similar a la controversia de Yamal Jashogyi, ${ }^{7}$ en el marco de una constante intromisión de las autoridades reales en el periodismo (García, 2007). Asímismo, las inversiones internacionales a pesar de ser cuantiosas, no han sido las esperadas por la corona, esto aunado a una histórica política autoritaria y hermética, pero también a megaproyectos tecnológicos anteriores fallidos. ${ }^{8}$

Por otro lado, la importancia geoestratégica que posee la zona es uno de los puntos en los que se debe hacer hincapié. Es necesario analizar esto, puesto que detrás de un proyecto futurista que busca generar una supuesta mayor independencia del sector fósil, existe también un fundamento económico ligado al Mar Rojo y al Canal de Suez, lo cual implica para Arabia Saudita entradas comerciales para una ruta que comprende un ciclo completo de capital, es decir, de producción, distribución y consumo.

En este sentido, las alianzas políticas que del proyecto NEOM se desprenden demuestran los esfuerzos por el Reino para configurar el equilibrio de poder y modificar el status quo de la península a su conveniencia. Esto debido a los intereses expansionistas y hegemónicos del RAS, los cuales no pueden materializarse a través de posesiones territoriales, pero pueden ser interpretados en los incentivos económicos que se otorgan a otros Estados con el fin de solventar sus crisis financieras.

\footnotetext{
Periodista de Washington Post, Yamal Jashogyi fue asesinado el 2 de octubre de 2018 por el gobierno saudí en el consulado de su país en Estambul. Como periodista disidente de la monarquía saudí, huyó del país en 2017 para continuar con sus artículos críticos en contra de Mohammad bin Salman y de Salmán bin Abdulaziz.

8 En particular, destaca el proyecto de 1976 cuando el reino intentó remolcar icebergs desde la Antártida para abastecer al país y cumplir con las necesidades hídricas. A pesar de la fuerte inversión, el proyecto fue cancelado y en su lugar se adoptó la desalinización. Además, resalta la Ciudad Económica Rey Abdullah, ubicada el norte de Yidda y de la próxima NEOM. Se trata de un núcleo urbano comenzado en 2005 y que aún no ha sido completado.
} 
Se observa entonces, que la narrativa desarrollista comprende a primera vista argumentos sociales y económicos. De este primer punto, tienen origen las grandes cantidades de inversión destinadas a ello, generando argumentos atractivos que demuestran la aceptación que la mayor parte de la población saudí otorga a la construcción y modificación del espacio que está destinado a ocupar. Sin embargo, a lo largo del presente ensayo se demuestra la poca viabilidad que representa la Ciudad en términos de sustentabilidad socioambiental, política y económica.

La producción de este tipo de espacio se puede observar en diversos lugares del mundo y a diferentes escalas. Así, este proyecto trae consigo importantes consecuencias coherentes con el capitalismo moderno: por un lado, se observa el desplazamiento y violencia al que se encuentra sometida la población local y por el otro, la modificación y expoliación de recursos en la zona para poder satisfacer las necesidades infraestructurales. Lo anterior, contrasta con la promesa y estilo de vida que la Ciudad NEOM vende: urbanismo ambiental, tecnología de punta y sociedades evolucionadas.

Por ello, se puede afirmar que dichos esfuerzos no proveen un beneficio extensivo hacia la población sino que simplemente buscan la perpetuación de los intereses estatales y las alianzas estratégicas que esto pueda traer consigo. El monopolio financiero que solo queda en manos de la corona y que, a pesar de que busca diversificarse a través de la entrada de empresas extranjeras, solo demuestra que los ganadores seguirán perteneciendo a la élite saudí, así como a los miembros de las corporaciones inversionistas del extranjero.

La resistencia de los afectados resulta de gran trascendencia en este punto. La organización, ya sea activa o pasiva, de las comunidades para hacer frente al evidente desinterés estatal pueden significar una articulación efectiva de sus demandas ya que ante el contexto de fuerte resonancia de las comunicaciones, las redes sociales, así como los medios de prensa internacionales, representan una vía de oportunidad para brindarles mayor representatividad, con miras a hacer justicia a su sociabilización. 
Sin embargo, la alternativa más segura y eficaz — pero a largo plazo-, sería la construcción de nuevas categorías epistémicas y ontológicas que reconfiguren cómo percibimos el mundo. En específico, modificar los pilares correspondientes a la modernidad que merman el desenvolvimiento del individuo y, por ende, cómo construimos y explicamos el espacio.

Se tiene que transformar a la racionalidad que pone al hombre y a la razón como el centro de las abstracciones en una relación más dialéctica y recíproca con su entorno; dejar de priorizar los procesos económico-estatales y darle más voz a otras formas de representación en diferentes escalas; negar la valoración utilitaria de la realidad derivada del sistema capitalista y poner en práctica un diálogo de saberes, procurando reconectar con valores más tradicionales de nuestra existencia, y, finalmente, abandonar a la ciencia y tecnología como ideologías automáticas que no incorporan análisis sociales, ambientales y políticos en su implementación. Lo anterior, podría acercarnos a concebir una nueva forma civilizatoria que no busque ciegamente el crecimiento económico, sino que tenga como prioridad satisfacer las necesidades básicas de la humanidad.

\section{Referencias}

Arab News. (16 de junio de 2019). How one man plans to tow an iceberg to the Arabian Gulf, https://www.arabnews.com/ node $/ 1542856 /$ middle-east.

Banco Mundial. (2019).PIB (US\$ a precios actuales)-Saudi Arabia. https://datos.bancomundial.org/indicador/NY.GDP.MKTP. $\mathrm{CD}$ ?locations=SA.

BBC News. (31 de octubre de 2017). Cómo será NEOM, la mega ciudad futurista con la que ArabiaSaudita quiere bajar su dependencia del petróleo. https://www.bbc.com/mundo/noticias-41809445.

BBC News. (2018). Jamal Khashoggi: 4 preguntas para entender el caso del periodista cuya muerte tiene en la mira a Arabia Saudita. https://www.bbc.com/mundo/noticias-internacional-45914541. 
Beck, U. (1998). La Sociedad del Riesgo. Hacia una nueva modernidad. Paidós Ibérica.

Berman, M. (2004). Todo lo sólido se desvanece en el aire. La experiencia de la modernidad. Siglo XXI.

British Petroleum. (2020). Statistical Review of World Energy 2020. https://www.bp.com/content/dam/bp/business-sites/en/global/ corporate/pdfs/energy-economics/statistical-review/bp-stats-review-2020-full-report.pdf?utm_source=BP_Global_GroupCommunications_UK_external\&utm_medium=email\&utm_campaign $=11599394$ Statistical\%20Review\%202020\%20-\%20 on\%20the\%20day\%20reminder\&dm_i=1PGC\%2C6WM5E\%2COV0LQ4\%2CRQC75\%2C1>.

Dembowski, H. (2018, 6 de marzo). Grand Neom vision is unrealistic, but politically useful. https://www.dandc.eu/en/blog/ grand-neom-vision-unrealistic-politically-useful.

Echeverría, B. (2018). Las ilusiones de la modernidad. Alacena Bolsillo.

Escobar, A. (2014). La invención del desarrollo. Universidad del Cauca.

García Suárez, P. (2007). La Prensa en Arabia Saudí: Cien Años de Vocación Internacional. Hesperia. Culturas del Mediterráneo, III(III), 13-28. https://books.google.com.mx/books?id=-Se_ $\mathrm{k} 2 \mathrm{Fs} 2 \mathrm{gQC} \&$ printsec $=$ frontcover\&dq=historia + de+arabia+saudita\&hl=es\&sa $=X \&$ redir_esc $=y \# \mathrm{v}=$ onepage $\& \mathrm{q}=$ historia\%20de\%20arabia\%20saudita\&f=false

Garduño, M. (2021). Arabia Saudita y las dinámicas de financiarización de Oriente Medio: algunas repercusiones geopolíticas del proyecto Neom en el Mar Rojo. Relaciones Internacionales 42, 141-256. https://revistas.uam.es/plugins/generic/ pdfJsViewer/pdf.js/web/vie...2Fdownload\%2FrelFrelacionesinternacion2019.42.008\%2F11455\%2F28309.

Garduño, M., (2019).La Rivalidad Geopolitica de Irán y Arabia Saudí en Medio Oriente. Universidad Nacional Autónoma de México. 
Habitat International Coalition. (2020, 2 de junio). Neom Megaproject. http://www.hlrn.org/img/violation/Neom2.pdf.

Henderson, S. (2019, 4 de febrero). Understanding the Saudi Aramco IPO. https://www.washingtoninstitute.org/policy-analysis/ understanding-saudi-aramco-ipo.

Holmes, F., (2019, 11 de noviembre), Should You Buy the Saudi Aramco IPO? https://www.forbes.com/sites/greatspeculations/2019/11/11/ should-you-buy-the-saudi-aramco-ipo/?sh=6a3571981c51.

Institut de Publique Sondage d'Opinion Secteur, (2019, enero). Saudi Arabia's Megacity: The NEOM Project. Overview OfPublic Perceptions Towards The Worlds Most Ambitious Project. https://www. ipsos.com/sites/default/files/ct/news/documents/2019-01/saudi_arabias_mega-city_-_the_neom_project.pdf.

KPMG. (2017, febrero). Saudi Arabia's Vision 2030 The Kingdom's plan for life after oil. https://assets.kpmg/content/dam/kpmg/ uk/pdf/2017/03/saudi-vision-2030-life-after-oil-kpmg-corporate-intelligence.pdf.

Lefebvre, H. (1974). La producción del espacio. Papers. Revista de Sociologia , 3, 1974.

Leff, E., (2010), Imaginarios Sociales y Sustentabilidad. http:// www.scielo.org.mx/pdf/crs/v5n9/v5n9a2.pdf.

Matthew, M., El Wardani, S. \& Abu Omar, A. (27 de enero de 2021). Saudi Arabia Aims to Become Next Germany of Renewable Energy.https://www.bloomberg.com/news/articles/2021-01-27/ saudi-arabia-aims-to-become-the-germany-of-renewable-energy

Moser, S.et.al. (2015). King Abdullah Economic City:Engineering Saudi Arabia's post-oil future. doi:10.1016/j.cities.2015.03.001 NEOM. (2021). NEOM. https://www.neom.com/en-us.

Osman, N. \& Abu Sneineh, M., (16 de abril de 2020). Saudi activist's killing exposes local tensions over Neom construction. https://www.middleeasteye.net/news/tribal-activist-reportedly-killed-protesting-saudi-neom-megacity-project.

Public Investment Fund, (2017). HRH the Crown Prince Mohammed bin Salman announces: NEOM-The Destination for the Future. https:/www.pif.gov.sa/en/MediaCenter/Pages/NewsDetails.aspx?NewsID=31. 
Rashed, I. (16 de agosto de 2018). NEOM: un nuevo canal para la cooperación egipcio-saudí. https://www.egypttoday.com/ Article/1/56096/NEOM-A-new-channel-for-Egyptian-Saudi-cooperation.

Reino de Arabia Saudita. (s.f.). Mensaje del principe Mohammed bin Salman bin Abdulaziz Al-Saud. https://www.vision2030. gov.sa/en/vision/crown-message.

Reino de Arabia Saudita, (s.f.). Visión 2030. https://www.vision2030.gov.sa/media/rc0b5oy1/saudi_vision203.pdf.

Rynhold J., \& Yaari, M. (2019). The quiet revolution in Saudi-Israeli relations, Mediterranean Politics. https://doi.org/10.108 0/13629395.2019.1699267.

Saudi Arabia Monetary Agency. (2019). 56th Annual Report. https://www.sama.gov.sa/en-US/EconomicReports/AnnualReport/Annual_Report_56th-EN.pdf. 